ARTICLE

\title{
Temporal dynamics of gene expression and histone marks at the Arabidopsis shoot meristem during flowering
}

Yuan You ${ }^{1, \dagger}$, Aneta Sawikowska ${ }^{2, \dagger}$, Manuela Neumann ${ }^{1}$, David Posé ${ }^{1, \dagger}$, Giovanna Capovilla', Tobias Langenecker ${ }^{1}$, Richard A. Neher ${ }^{3, \dagger}$, Paweł Krajewski ${ }^{2} \&$ Markus Schmid ${ }^{1,4}$

Plants can produce organs throughout their entire life from pluripotent stem cells located at their growing tip, the shoot apical meristem (SAM). At the time of flowering, the SAM of Arabidopsis thaliana switches fate and starts producing flowers instead of leaves. Correct timing of flowering in part determines reproductive success, and is therefore under environmental and endogenous control. How epigenetic regulation contributes to the floral transition has eluded analysis so far, mostly because of the poor accessibility of the SAM. Here we report the temporal dynamics of the chromatin modifications H3K4me3 and H3K27me3 and their correlation with transcriptional changes at the SAM in response to photoperiod-induced flowering. Emphasizing the importance of tissue-specific epigenomic analyses we detect enrichments of chromatin states in the SAM that were not apparent in whole seedlings. Furthermore, our results suggest that regulation of translation might be involved in adjusting meristem function during the induction of flowering.

\footnotetext{
${ }^{1}$ Department of Molecular Biology, Max Planck Institute for Developmental Biology, Spemannstrasse 35, 72076 Tübingen, Germany. ${ }^{2}$ Department of Biometry and Bioinformatics, Institute of Plant Genetics, Polish Academy of Sciences, Strzeszyńska 34, 60-479 Poznań, Poland. ${ }^{3}$ Evolutionary Dynamics and Biophysics Group, Max Planck Institute for Developmental Biology, Spemannstrasse 35, 72076 Tübingen, Germany. ${ }^{4}$ Umeå Plant Science Centre, Department of Plant Physiology, Umeå University, SE-901 87 Umeå, Sweden. †Present address(es): Zentrum für Molekularbiologie der Pflanzen, EberhardKarls-Universität Tübingen, Auf der Morgenstelle 32, Tübingen D-72076, Germany (Y.Y.); Department of Statistical and Mathematical Methods, Poznań University of Life Sciences, Wojska Polskiego 28, 60-637 Poznań, Poland (A.S.); Facultad de Ciencias, Departamento de Biología Molecular y Bioquímica, Instituto de Hortofruticultura Subtropical y Mediterránea, Universidad de Málaga-Consejo Superior de Investigaciones Científicas, Universidad de Málaga, 29071 Málaga, Spain (D.P.); Biozentrum, University of Basel, Klingelbergstrasse 50/70, CH-4056 Basel, Switzerland (R.A.N.). Correspondence and requests for materials should be addressed to M.S. (email: markus.schmid@umu.se).
} 
$\mathrm{n}$ contrast to most animals, plants have the capacity to produce new organs due to the life-long maintenance of populations of pluripotent stem cell in specialized reservoirs called shoot apical meristems (SAM), from which the aerial parts of a plant, stems, leaves and flowers, are derived ${ }^{1}$. This endows plants with the ability for continued growth, and also allows them to adjust rapidly and flexibly to changes in their environment, which is of particular importance considering the sessile life style of plants ${ }^{2}$. At the time of flowering, the SAM of Arabidopsis thaliana undergoes a remarkable phase transition and becomes an inflorescence meristem (IM) that induces on its flanks flower meristems, from which floral organs will differentiate (Supplementary Fig. 1a). At the same time the central IM needs to remain in an undifferentiated state to ensure continued growth ${ }^{3}$.

The correct timing of flowering is crucial to ensure reproductive success and is therefore of both adaptive and economic value ${ }^{2}$. Genetic analyses have identified a number of molecular pathways that perceive and integrate diverse endogenous and environmental signals to ensure that flowering commences under the most favourable conditions ${ }^{2,4}$. Prominent examples are the vernalization pathway that regulates flowering in response to prolonged cold (overwintering) and the photoperiod pathway that in A. thaliana promotes flowering in long-day (LD) conditions. Day length is perceived in the leaves and through a complex regulatory mechanisms that involves light signalling and the circadian clock ${ }^{2}$ results in the stable expression of the transcription factor (TF) CONSTANS (CO) protein specifically at the end of the LD. CO in turn activates the expression of FLOWERING LOCUS T (FT) and TWIN SISTER OF FT (TSF) in the phloem companion cells of the leaf vasculature $^{5,6}$. The FT and TSF proteins then act as a longrange signal (florigen) that transduces the information to induce flowering from the leaf to the SAM where they interact with the bZIP TF FD to activate expression of flowering time and floral homeotic genes such as the MADS-box TFs SUPPRESSOR OF OVEREXPRESSION OF CO 1 (SOC1) and APETALA 1 (AP1) $)^{7}$.

Most of the known floral regulator genes encode TFs or transcriptional co-regulators that form a complex genetic circuit to ensure flowering is initiated under the right circumstances. While we understand the function of many of the individual genes in this circuit relatively well, genome-wide analyses have been complicated by the fact that flowering time signals are integrated in specific cell types such as the phloem companion cells in leaves and the SAM. Several studies have investigated the transcriptional changes during the initiation of flowering and early flower development at the SAM by either hand dissection or laser-capture micro-dissection of meristems, followed by transcription profiling using either microarrays or RNA-seq ${ }^{8-10}$. These studies provided insights into the changes in expression at the SAM during the floral transition in a tissue-specific manner and identified previously uncharacterized genes with a potential role in flowering time regulation. However, samples generated by these methods are not well suited for epigenomic analyses as they generally contain only small amounts of chromatin. This is unfortunate, as epigenetic factors clearly play an important role in flowering time control ${ }^{11}$. For example, expression of the important flowering time integrator genes $F T^{12,13}$ in the phloem companion cells of leaves and FLOWERING LOCUS C $(F L C)^{14}$ in response to vernalization have both been shown to be controlled in part at the chromatin level. However, these studies have been performed on complex tissues or whole seedlings and little is known about the epigenetic processes that regulate flowering time locally, mostly because of the poor accessibility of tissues such as the SAM. It is challenging to isolate enough high quality SAM samples for multiple study approaches in spatiotemporal developmental programs.
To overcome this limitation we have adopted INTACT, which employs in vivo biotinylation of nuclear envelopes to facilitate purification of nuclei from specific tissues ${ }^{15}$, to describe the temporal dynamics of the chromatin modifications H3K4me3 and $\mathrm{H} 3 \mathrm{~K} 27 \mathrm{me} 3$, which have been implicated in promoting and repressing gene expression, respectively ${ }^{16,17}$, and their correlation with transcriptional changes at the SAM during photoperiodinduced flowering. Emphasizing the importance of tissue-specific analyses we observe narrow peaks of $\mathrm{H} 3 \mathrm{~K} 27 \mathrm{me} 3$ close to or even covering the transcription start sites (TSS) of thousands of loci, a chromatin feature rarely observed in epigenomic studies conducted on complex tissues. Our results also suggest that control of translation could be an important factor in regulating meristem function during the induction of flowering.

\section{Results}

Establishment of INTACT for the SAM. In order to study transcriptional responses and the dynamic of epigenetic modifications at the shoot meristem during the floral transition we first established a modified INTACT reporter line that employs two promoters to biotinylate nuclear envelopes in the entire SAM and IM (Fig. 1a,b and Supplementary Fig. 1c; Methods section $\left.^{15,17}\right)$. Enrichment of SAM marker genes such as SHOOT MERISTEMLESS (STM), CLAVATA3 (CLV3) and FD in nuclei isolated from the INTACT line was verified by semi-quantitative RT-PCR (Fig. 1c, Supplementary Figs 1e and 2). Synchronous flowering of the INTACT reporter line was accomplished by shifting 21-day-old plants from non-inductive short days (SD) to inductive LD conditions (Supplementary Fig. 1b). Two to three days after the shift, we consistently detected by RNA in situ hybridization robust expression of the flower marker gene AP1, indicating floral commitment (Fig. 1d,e and Supplementary Fig. 1d). Furthermore, quantitative reverse transcription PCR (qRT-PCR) using nuclear RNA isolated from meristems of plants transferred from SD to LD demonstrated a significant increase in the expression of the meristematic flowering time integrators SOC1 and AGAMOUS-LIKE 24 (AGL24) within $24 \mathrm{~h}$ after the shift (Fig. 1f). Taken together these initial results indicate that our INTACT line constitutes a system suitable for the study of photoperiod-controlled floral transition at the SAM.
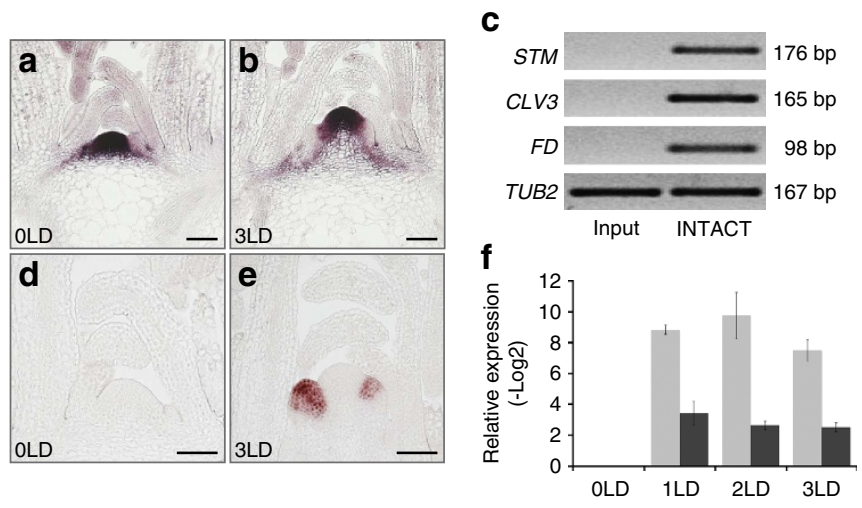

Figure 1 | Establishment of the INTACT system for the meristem. $(\mathbf{a}, \mathbf{b})$ Detection of biotinylation and $(\mathbf{d}, \mathbf{e})$ expression of the floral marker gene AP1 at the meristem of the INTACT line before $(\mathbf{a}, \mathbf{d})$ and 3 days after $(\mathbf{b}, \mathbf{e})$ the shift to LD. Scale bar, $50 \mu \mathrm{m}$. (c) Semi-quantitative RT-PCR detecting expression of STM, CLV3 and FD in the total nuclei from the samples enriched for shoot apical region by manual dissection (input) and in the nuclei isolated by INTACT from the meristem (INTACT). (f) Quantitative RT-PCR of SOC1 (light grey) and AGL24 (dark grey) in the nuclei from the meristem before (0), and 1,2 and 3 days after the shift to LD. Error bars, values of two biological replications. 
Differential gene expression at the SAM during flowering. Having established INTACT for the SAM we next performed RNA-seq of the nuclear RNA pool isolated from the meristem for four biological replicates $0,1,2$ and 3 days after the shift to LD. In total we detected expression (FPKM $>1$ ) of $\sim 60 \%(19,940)$ of all genes annotated in Arabidopsis thaliana at the SAM at least at one time point (Supplementary Data 1), which is comparable to previous reports ${ }^{8,9}$. The meristem identity genes ${ }^{18,19}$, STM, KNOTTED-LIKE FROM ARABIDOPSIS THALIANA 1 (KNAT1), CLAVATA 1 (CLV1) and CLV3 were stably detected in all samples (Fig. 2a), indicating the presence of mRNA originating from meristems and confirming results from the semi-quantitative RT-PCR analysis (Fig. 1c). In contrast, the early floral homeotic genes ${ }^{20}$ AP1, APETALA 3 (AP3) and CAULIFLOWER $(C A L)$, and genes expressed in the centre or abaxial side of developing leaves ${ }^{21}$ such as JAGGED (JAG), WUSCHEL RELATED HOMEOBOX 1 (WOX1) and WOX3, were basically undetectable (Fig. 2b,c), indicating strong enrichment for mRNA from the SAM.

Pairwise comparison at the four time points identified 298 significantly differentially expressed genes, including 148 and 146 genes that were consistently up- or down-regulated, respectively, and 4 genes that exhibited more complex expression patterns (Supplementary Data 2). Interestingly, expression of the morning-expressed genes of the central oscillator of the circadian clock $^{22}$ LATE ELONGATED HYPOCOTYL (LHY) and CIRCADIAN CLOCK ASSOCIATED 1 (CCA1) was increased significantly 1 day after the shift to LD (Fig. 2d). In contrast, the evening-expressed clock gene ${ }^{22}$ PSEUDO-RESPONSE REGULATOR 5 (PRR5) was significantly down-regulated. LUX ARRHYTHMO (LUX) and EARLY FLOWERING 4 (ELF4), which encode components of the evening complex, displayed a similar trend as PRR5, but the expression changes were not statistically significant after correction for multiple testing (Fig. 2d). These rapid changes in transcript levels most likely reflect a change in the phase of clock gene expression in response to the shift from $\mathrm{SD}$ to LD rather than a change in amplitude ${ }^{23}$. Increased expression following the shift to LD was also detected for SOC1, $F D$ and the CCAAT-binding TF NF-YA4, which are known to promote flowering 24 (Fig. 2e). The expression changes determined by RNA-seq for SOC1 confirm previous results from qRT-PCR experiments (Fig. 1f), emphasizing the reproducibility of the INTACT method. In addition to induction of floral promoters we also observed decreased expression for the flowering-time repressor JMJ30 (ref. 25; Fig. 2e). Interestingly, expression of SHEPHERD (SHD), which is required for stem cell functions and affects the expression of $C L V 3$ and WUSCHEL (WUS) and is responsible for the

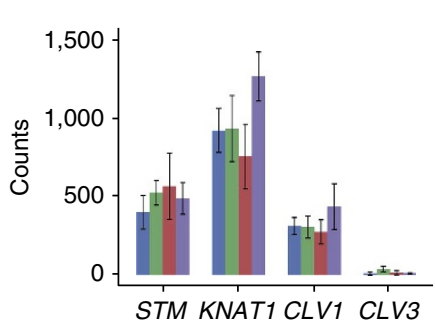

e

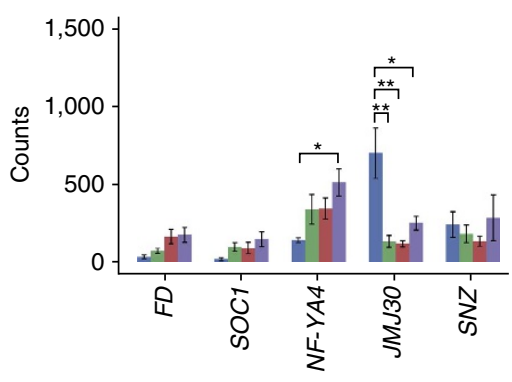

b

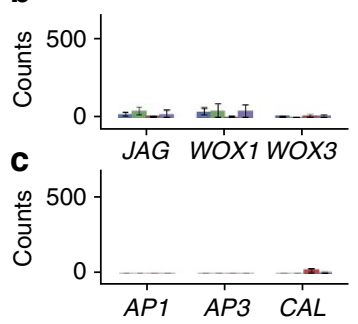

d

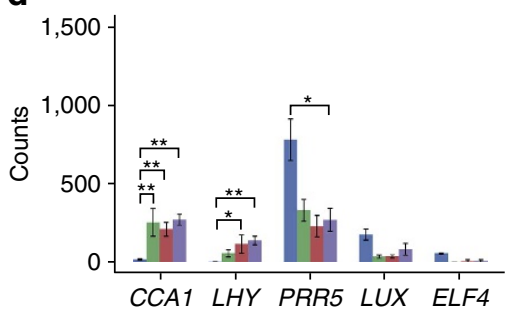

h

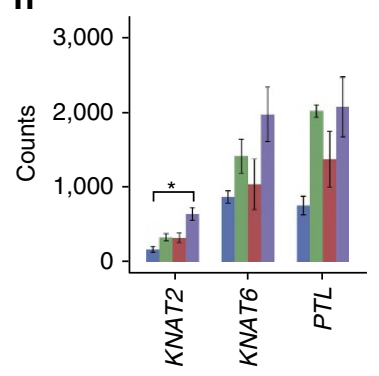

f

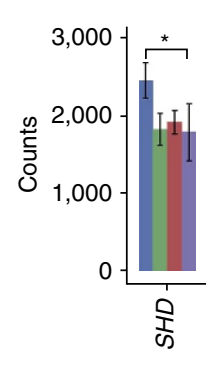

g

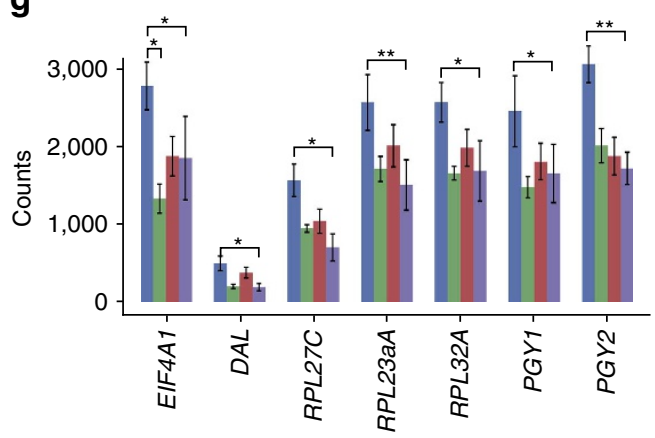

i $\quad>6 \quad 4 \quad 2 \quad 0 \quad 2 \quad 4>6$

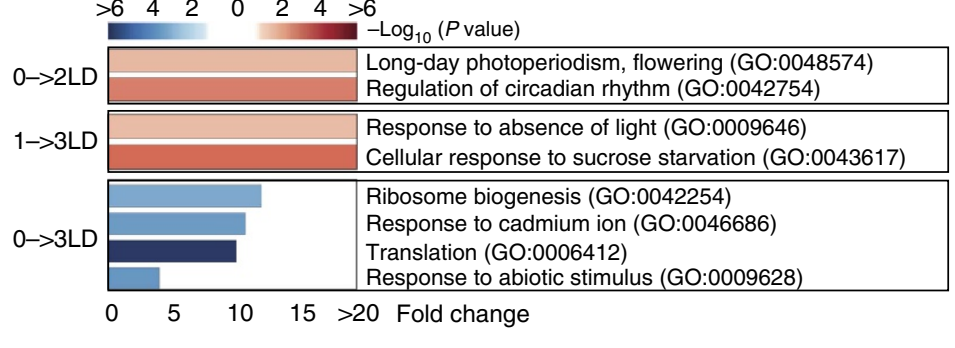

Figure 2 | Differential gene expression in the meristem during flowering. (a-h) Expression (counts of reads) of marker genes for the meristem (a), leaf primordia (b), flower primordia (c), circadian clock (d), flowering time (e), stem cell (f), translation ( $\mathbf{g}$ ) and lateral organ boundary (h) at the SAM before (blue), and 1 (green), 2 (red) and 3 (purple) days after the shift to LD. Error bars, \pm s.e. based on four biological replicates; ${ }^{\star} P<0.05,{ }^{\star} P<0.01$ (Deseq2 $\mathrm{P}$ values corrected for multiple testing using the Benjamini-Hochberg method). (i) Biological GO functions enriched for differentially regulated genes with increased (red) and decreased (blue) expression. Only significantly enriched GO terms $(P<0.05)$ are reported. 
formation of functional CLAVATA proteins $^{26}$, was also significantly decreased upon shift to LD (Fig. 2f). The shd mutant is characterized by an increase in stem cell number and an enlarged meristem ${ }^{26}$ and a decrease in SHD expression would thus be in agreement with the growth of the meristem that accompanies the floral transition ${ }^{3}$. Taken together these findings indicate that it is possible to capture changes in gene expression of important flowering time and meristem identity genes in the nuclear RNA pool isolated by INTACT from the SAM.

Gene ontology (GO) analysis confirmed that genes related to long-day photoperiodism (GO:0048574) and regulation of circadian rhythm (GO:0042754) were significantly overrepresented among the genes induced at the SAM (Fig. 2i and Supplementary Data 3). In contrast, down-regulated transcripts were significantly enriched for genes related to ribosome biogenesis (GO:0042254) and the control of translation (GO:0006412; Fig. 2i). Affected genes include the translation initiation factor EIF4A1, the ribosomal RNA (rRNA) processing factor DIFFERENTIATION AND GREENING-LIKE $(D A L)^{27}$, and the ribosomal structural constituents PIGGYBACK 1 (PGY1), $P G Y 2$ and RPL23aA (Fig. 2g, Table 1). Interestingly, mutations in the PGY genes and RPL23aA have previously been shown to cause (or enhance) developmental phenotypes including defects in leaf polarity and inflorescence architecture, and late flowering ${ }^{28-30}$. Taking together these results suggest a connection between the control of translation and the regulation of developmental phase transition at the SAM.

Changes in H3K4me3 at the SAM correlate with gene expression. To study the correlation between gene expression and chromatin features during floral transition we next analysed the genome- wide distribution of tri-methylation on lysine 4 or 27 of histone 3 (H3), epigenetic marks that are often associated with transcriptional activation (H3K4me3) and repression (H3K27me3), respectively ${ }^{16,17}$. ChIP-seq was performed on nuclei isolated from the SAM at the four time points for two biological replicates using $\mathrm{H} 3 \mathrm{~K} 4 \mathrm{me} 3$ - and H3K27me3-specific antibodies (Supplementary Fig. 3).

H3K4me3 was detected on $61.6 \%(16,746)$ of protein-coding genes at least at one time point, and was mostly $(81.5 \% ; 13,647)$ associated with the TSS (Fig. 3a, Supplementary Fig. 4a and Supplementary Data 4). We observed a significant positive association between the presence of H3K4me3 on the TSS and gene expression, as (on average over four time points) the proportion of genes marked with $\mathrm{H} 3 \mathrm{~K} 4 \mathrm{me} 3$ on the TSS was $5.02 \% \quad( \pm 0.31 \%$; \pm refers to s.e., $n=4)$ and $66.13 \%$ ( $\pm 1.68 ; \pm$ refers to standard error, $n=4$ ) for the non-expressed and expressed genes $(\mathrm{FPKM}>1)$, respectively (Fig. $3 \mathrm{~b}$ and Supplementary Fig. 5b). Furthermore, for genes expressed at a given time point, the level of $\mathrm{H} 3 \mathrm{~K} 4 \mathrm{me} 3$ signal on the TSS was significantly (Spearman rank correlation ranging from 0.29 to 0.33 over the four time points, $P<0.001$, test based on $t$ approximation) correlated with the level of gene expression (Fig. 3a and Supplementary Fig. 5a). For example, H3K4me3 was significantly enriched at the TSS of the SAM marker gene STM in the SAM samples (Fig. 4d). Over the course of the floral induction, 2,024 genes displayed significant changes in $\mathrm{H} 3 \mathrm{~K} 4 \mathrm{me} 3$ signal over the gene body at the SAM (Supplementary Data 5). The levels of H3K4me3 changes were positively correlated with the significance of gene expression changes (Fig. $3 \mathrm{c}$ and Supplementary Fig. $4 \mathrm{~b}$ ), and 56 genes changed significantly both in $\mathrm{H} 3 \mathrm{~K} 4 \mathrm{me} 3$ signal and in expression, mostly $(89.3 \%)$ in the same direction (Supplementary Fig. 6a). The mean expression of genes that increased in $\mathrm{H} 3 \mathrm{~K} 4 \mathrm{me} 3$ upon shift from SD to LD (pairwise

Table 1 | Significantly differentially expressed structural constituents of ribosome and genes for RNA-binding proteins.

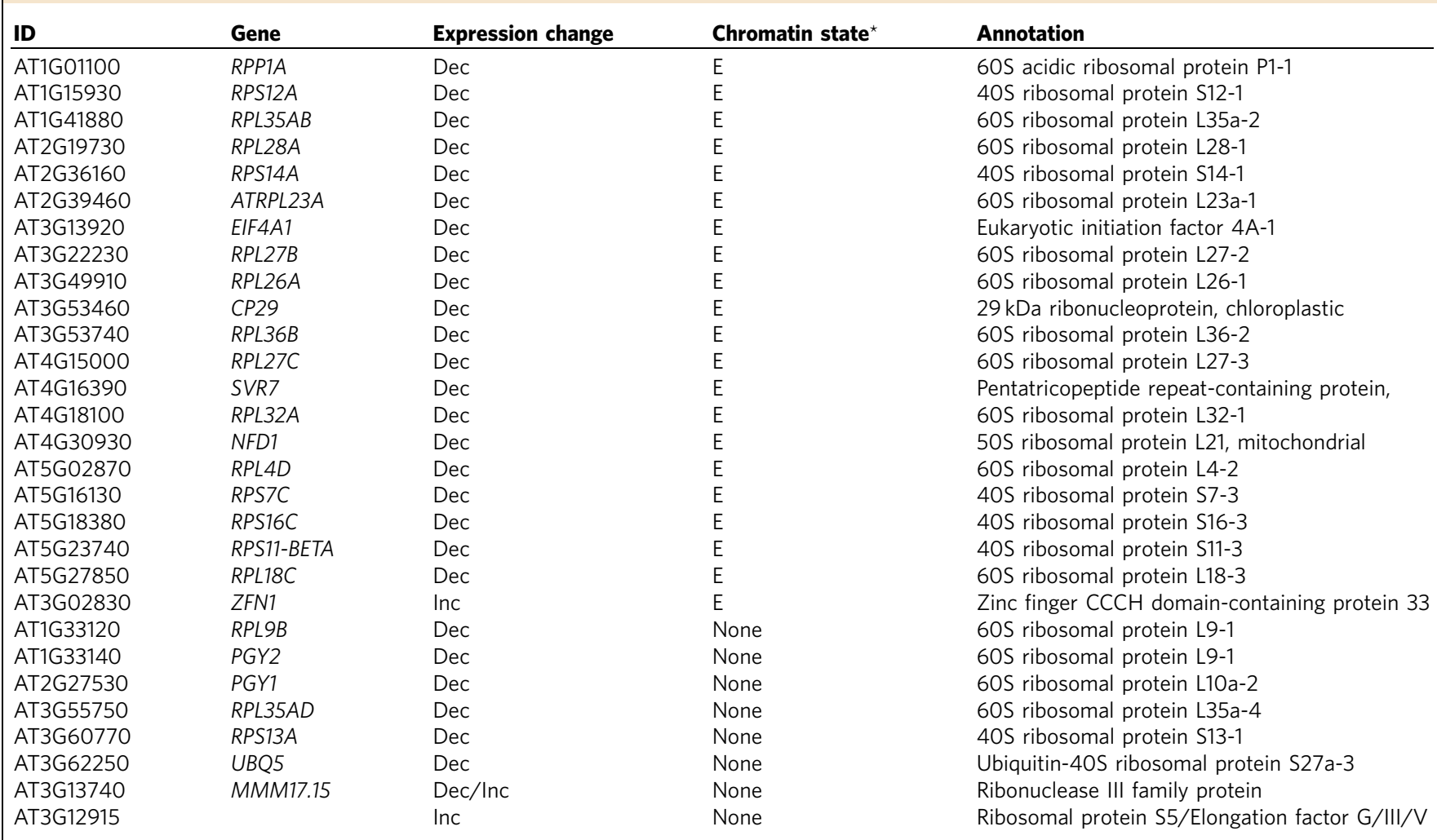

${ }^{\star}$ Genes that were in $\mathrm{H}$-state, E-state (E) or none of the two (None) at least at one time point. 
a
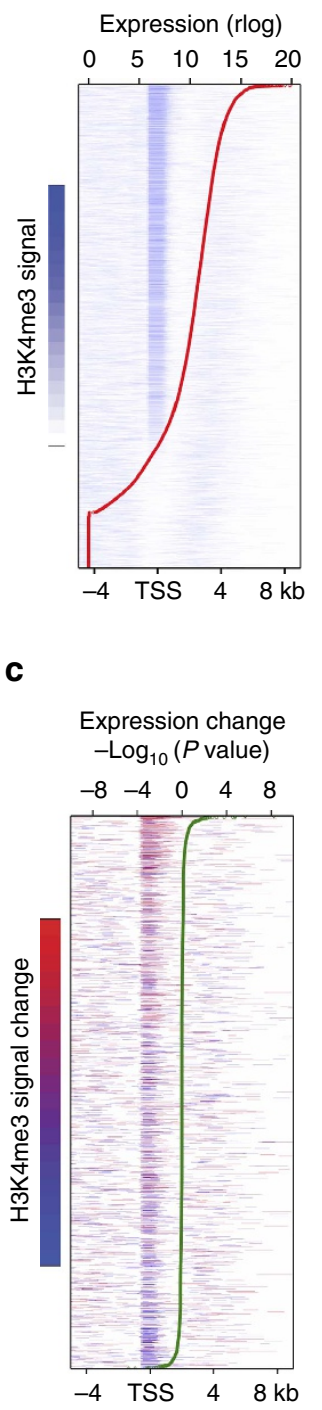

b

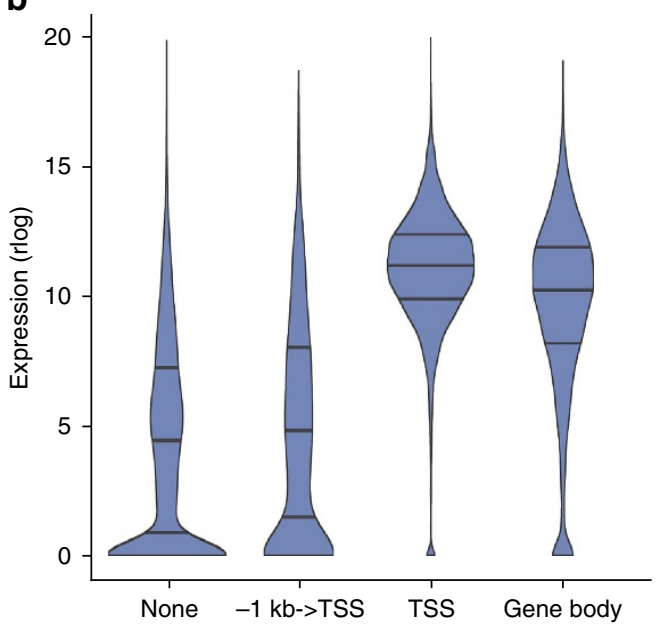

d

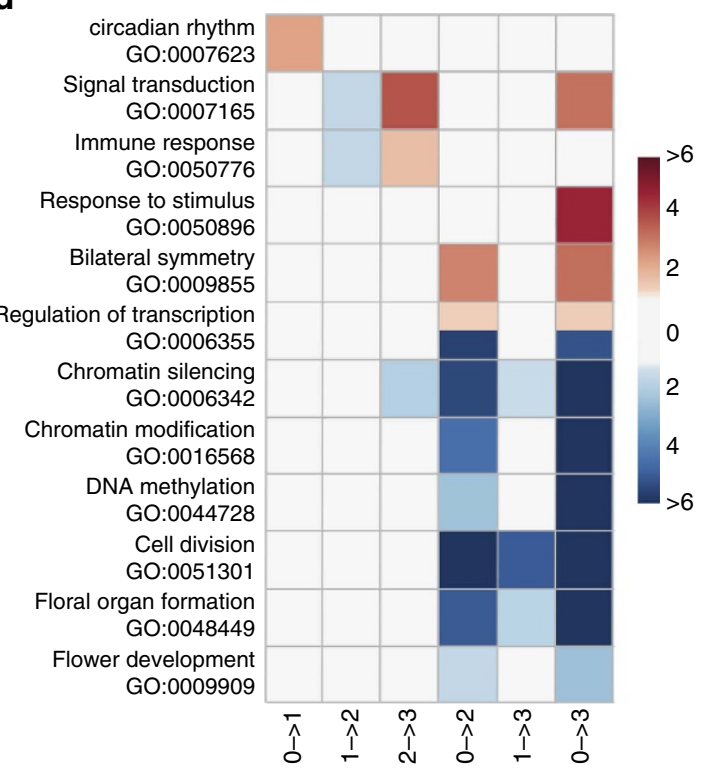

Figure 3 | Positive correlation between H3K4me3 and expression. (a) Normalized H3K4me3 signal on protein-coding genes in 0 LD samples between $-4 \mathrm{~kb}$ and $+8 \mathrm{~kb}$ around the TSS. Genes ordered according to their expression level (regularized logarithm (rlog) of counts, red line). (b) Distribution of expression of protein-coding genes in 0 LD samples in relation to the position of the H3K4me3 marks. (c) Protein-coding genes with significant differences in H3K4me3 signal between $\mathrm{OLD}$ and 3 LD samples. Red and blue indicate the direction and significance of gain and loss of H3K4me3, respectively. Genes ordered according to significance ( $-\log _{10}(P$ value)) of changes in expression (green line). (d) Key biological processes enriched in genes with significant H3K4me3 changes. Colour key indicates the significance ( $-\log _{10}(P$ value)) of the enriched GO terms that gained (red) and lost (blue) H3K4me3, respectively. Only significantly enriched GO terms $(P<0.05$; fold-change $>2)$ are shown.

comparisons: $0->1 ; 0->2$; and $0->3$ ) increased significantly $(P<0.05$; test based on rejecting zero slope of linear regression of mean expression against time; Supplementary Fig. 6b), whereas the expression changes observed in the remaining comparisons, while generally following the same trend, were not significant. In summary, our findings suggest a general congruence between $\mathrm{H} 3 \mathrm{~K} 4 \mathrm{me} 3$ dynamics and gene expression.

GO analysis of the 2,024 genes that exhibited significant changes in $\mathrm{H} 3 \mathrm{~K} 4 \mathrm{me} 3$ identified 12 overrepresented biological processes (Fig. 3d, Supplementary Data 6). For example, H3K4me3 changed within $24 \mathrm{~h}$ after the shift to LDs on genes that form the central oscillator of the circadian clock, as also seen at the transcript level (Fig. 2d), followed by signal receptors, TFs (Supplementary Fig. 7), chromatin remodelers, and categories which play important regulatory roles in cell division, differentiation and developmental phase transitions (Fig. 3d, Supplementary Data 6). Together these data establish changes in H3K4me3 abundance as a fast and important response in the regulation of genes at the SAM during the transition to flowering.

H3K27me3 is a weak predictor of gene expression at the SAM. Opposing the effect of $\mathrm{H} 3 \mathrm{~K} 4 \mathrm{me} 3$, which is often enriched at the TSSs of genes and promotes expression (Fig. 3a,b and Supplementary Fig. 5), is $\mathrm{H} 3 \mathrm{~K} 27 \mathrm{me} 3$, a canonical repressive epigenetic mark ${ }^{16,17}$. At the SAM H3K27me3 was detected on $63.69 \%(17,328)$ of protein-coding loci at least at one time point (Fig. 5a and Supplementary Data 7). This is in contrast to reports from analyses of complex tissues or whole seedlings, which had reported $\mathrm{H} 3 \mathrm{~K} 27 \mathrm{me} 3$ on less than $10,000 \operatorname{loci}^{31}$. In agreement with published findings ${ }^{16,17}$ broad regions of $\mathrm{H} 3 \mathrm{~K} 27 \mathrm{me} 3$ were 


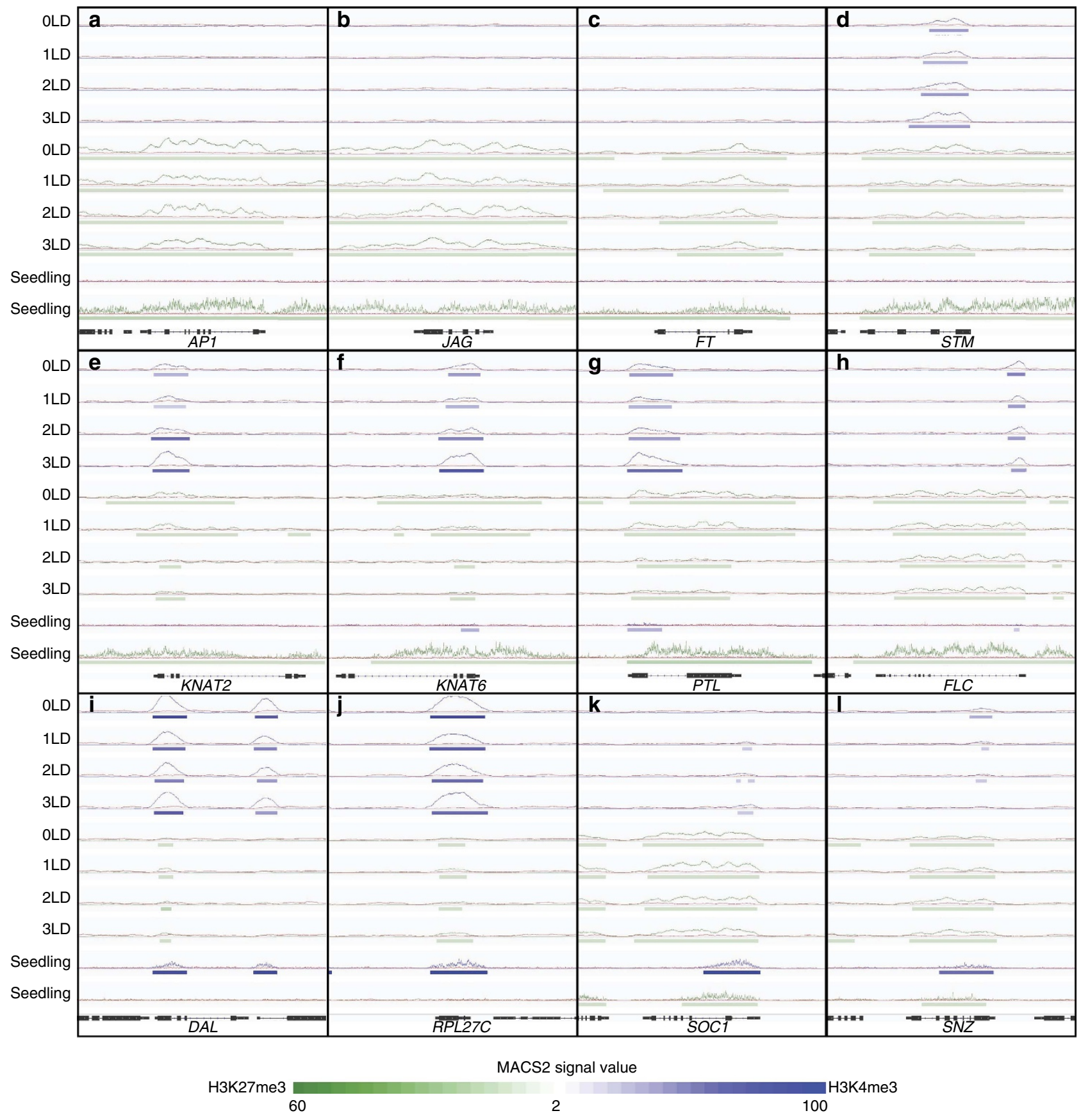

Figure 4 | Examples of H3K4me3 and H3K27me3 dynamics at the SAM during the floral transition. (a-i) Integrative Genomics Viewer traces of AP1 (a), JAG (b), FT (c), STM (d) KNAT2 (e), KNAT6 (f), PTL (g), FLC (h), DAL (i), RPL27C (j), SOC1 (k), SNZ (I). H3K4me3, H3K27me3 and H3 signals are depicted in blue, green and red, respectively. Horizontal bars indicate regions and signals of significant enrichment of H3K4me3 and H3K27me3 (according to analysis in MACS2).

observed on $40.56 \% \pm 0.95 \%$ (average over the four time points; \pm refers to s.e., $n=4)$ transcriptionally silent genes (FPKM $<1$, for example Fig. $4 \mathrm{a}-\mathrm{c})$. In addition, we also detected an enrichment of $\mathrm{H} 3 \mathrm{~K} 27 \mathrm{me} 3$ on the TSS of $15.84 \% \pm 0.93 \%$ (average over the four time points; \pm refers to s.e., $n=4$ ) of expressed genes in the SAM (Fig. 5a,b, and Supplementary Fig. 8a,b). The fraction of non-expressed genes $(\mathrm{FPKM}<1)$ was increased in the group of genes carrying H3K27m 3 mark on the TSS when compared to the other groups (Fig. 5b, Supplementary Fig. 8b). For expressed genes (FPKM $>1$ ), the H3K27me3 signal at the TSS was formally negatively correlated with the level of gene expression, though the Spearman rank correlation coefficient was rather low (0.07-0.08 over the four time points, $P<0.001$, test based on $t$ approximation). Changes in H3K27me3 at the TSS were not significantly correlated with changes in gene expression between time points (Fig. 5c and Supplementary Fig. 8c). The 472 genes that displayed significant changes in H3K27me3 signal over the gene body during the course of the floral transition (Supplementary Data 8) were not enriched for any biological and molecular functions in GO analysis.

Previous studies had suggested a negative correlation between gene expression level and H3K27me3 abundance ${ }^{31,32}$. However, our data indicate that during the transition to flowering changes in gene expression at the SAM are not always reflected in changes 
a

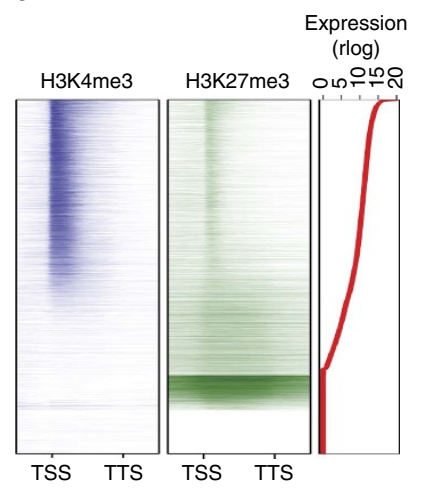

b

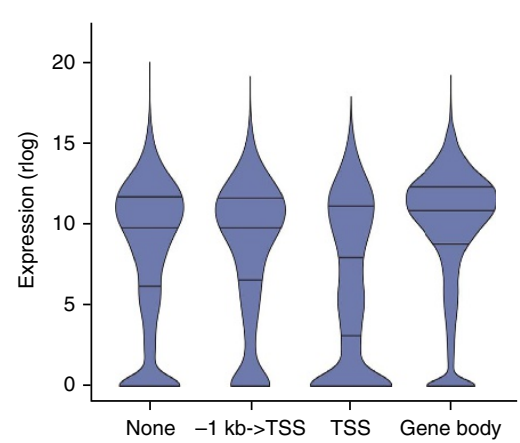

C

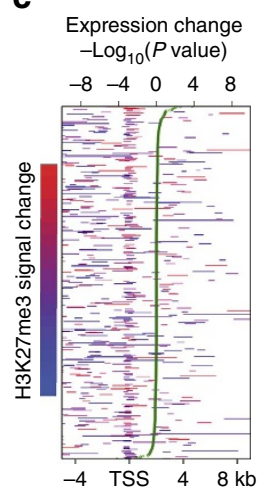

Figure 5 | Profiles of H3K4me3 and H3K27me3 marks at the SAM. (a) Distribution of normalized H3K4me3 and H3K27me3 signal across the body $( \pm 1 \mathrm{~kb})$ of protein-coding genes in 0 LD sample. Regions upstream of the TSS ( $-1 \mathrm{~kb})$ and downstream of TTS ( $+1 \mathrm{~kb})$ were each divided into 10 bins of length $100 \mathrm{bp}$. Gene bodies were normalized to account for differences in gene length and divided into 50 bins of equal width. Genes sorted based on levels of expression (red line), and genes with no detectable expression were sorted by H3K27me3 levels. (b) Distribution of expression of protein-coding genes in 0 LD samples in relation to the position of the H3K4me27 marks. (c) Protein-coding genes with significant differences in H3K27me3 signal between 0 and 3 LDs samples. Red and blue indicate the direction and significance of gain and loss of H3K27me3, respectively. Genes ordered according to significance $\left(-\log _{10}(P\right.$ value $)$ ) of changes in expression (green line).

in $\mathrm{H} 3 \mathrm{~K} 27 \mathrm{me} 3$ signals (Fig. 4). For example, the activation of SOC1 expression in response to LD is accompanied by deposition of $\mathrm{H} 3 \mathrm{~K} 4 \mathrm{me} 3$ mark whereas $\mathrm{H} 3 \mathrm{~K} 27$ me3 levels remain essentially unchanged (Fig. 4k).

Opposing H3K4me3-H3K27me3 modification states at the SAM. Overlapping regions of $\mathrm{H} 3 \mathrm{~K} 27 \mathrm{me} 3$ and $\mathrm{H} 3 \mathrm{~K} 4 \mathrm{me} 3$ have been described in plants before, both on individual genes as well as in genome-wide analyse ${ }^{17,33,34}$. In the meristem, co-occurrence of $\mathrm{H} 3 \mathrm{~K} 4 \mathrm{me} 3$ and $\mathrm{H} 3 \mathrm{~K} 27 \mathrm{me} 3$ was mainly detected within $\pm 500 \mathrm{bp}$ around the TSS of expressed genes (Fig. 5a; Supplementary Fig. 9a), and two categories of H3K4me3H3K27me3 states could be distinguished (Fig. 6c,d; Supplementary Fig. 9b, Supplementary Fig. 10, Supplementary Data 9): broad $\mathrm{H} 3 \mathrm{~K} 27 \mathrm{me} 3$ regions containing a narrow H3K4me3 peak around the TSS $( \pm 500 \mathrm{bp})$, to which we refer as 'harbouring' state (H-state); and an 'embedded' state (E-state) with opposite characteristics, that is, narrow $\mathrm{H} 3 \mathrm{~K} 27 \mathrm{me} 3$ peaks within broader $\mathrm{H} 3 \mathrm{~K} 4 \mathrm{me} 3$ domains. $28.3 \%$ of regions that displayed $\mathrm{H}$-state characteristics in the SAM were also detected in a control data set obtained by performing ChIP-seq in whole seedlings (Fig. 6a,c; Supplementary Data 10). In contrast, the embedded chromatin state was almost exclusively detected in INTACT samples obtained from the SAM and was rarely detected in whole seedlings, demonstrating the power of tissue-specific epigenomic studies (Fig. 6b,d; Supplementary Data 10).

Functions and dynamics of $\mathrm{H}$ - and $\mathrm{E}$ - chromatin state genes. To gain a better understanding of the potential differences in molecular functions of the $\mathrm{H}$ - and E- chromatin states at the TSS we performed GO enrichment analyses (Supplementary Data 11). We found that genes with TF (GO:0003700) and DNA binding activity (GO:0003677) were overrepresented among the genes that were in $\mathrm{H}$-state (Fig. 7a). Over the course of floral induction about half of the $\mathrm{H}$-states present in the vegetative meristem resolved into non-overlapping, partial overlapping or E-states, respectively, demonstrating the dynamic nature of this chromatin state (Fig. 7b). Genes include, for example, the homeobox TFs KNAT2 and KNAT6, and the trihelix TF PTL that are involved in meristem and lateral organ boundary development ${ }^{35-37}$. We observed that during floral transition H3K4me3 signal levels for these genes increased, while H3K27me3 domains decreased in length, and that these epigenetic changes were accompanied by increased expression (Fig. $2 \mathrm{~h}$ ). At the end of the floral transition the $\mathrm{H}$-state of $P T L$ became partial overlapping (H3K27me3 $>\mathrm{H} 3 \mathrm{~K} 4 \mathrm{me} 3)$, and KNAT2 and KNAT6 had converted to E-state (Fig. $4 \mathrm{e}-\mathrm{g}$ ).

In contrast, GO term analysis revealed that structural constituents of ribosome (GO:0003735), involved in translation (GO:00081345) and genes that encode for RNA-binding proteins (GO:0003726; GO:0044822) were overrepresented among the E-state genes (Fig. 7a). In this context it is worth remembering that genes involved in ribosome biogenesis, rRNA processing, and in the initiation of translation were significantly enriched among the genes whose expression in the SAM decreased during the transition to flowering (Table 1 and Fig. 2i).

Interestingly, several ribosomal protein genes were reported to express at the highest levels in meristems and young dividing tissues and at the lowest in non-dividing tissues, and mutants of these r-protein genes exhibit multiple developmental lethal/defect phenotypes ${ }^{38-42}$. Our result suggested that regulation at the translation level might play important roles for fate decision of the IM, however changes in expression of these translation factors are unrelated with $\mathrm{H} 3 \mathrm{~K} 4 \mathrm{me} 3$ and $\mathrm{H} 3 \mathrm{~K} 27 \mathrm{me} 3$ modification levels. Overall, the E-chromatin state was mostly stable (Figs 4i,j and 7b).

\section{Discussion}

Meristems are of interest to plant developmental biology as they harbour stem cells that divide slowly and give rise to daughter cells that eventually differentiate into all other cell types. Despite their importance it is still unclear what exactly distinguishes undifferentiated meristem cells from their differentiated descendants. It seems likely that regulation at the epigenetic and chromatin level contributes to the differentiation of cells derived from the meristem and there is mounting evidence that different cell types exhibit distinct epigenetic features. For example, unique DNA-methylation patterns have recently been reported for different cell types in the root apical meristem ${ }^{43}$. How other epigenetic marks such as acetylation or methylation of histones contribute to the function of meristems and how dynamic these modifications respond to change in the environment is, however, poorly understood. 
a

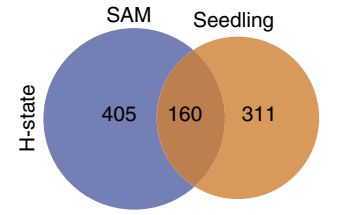

C

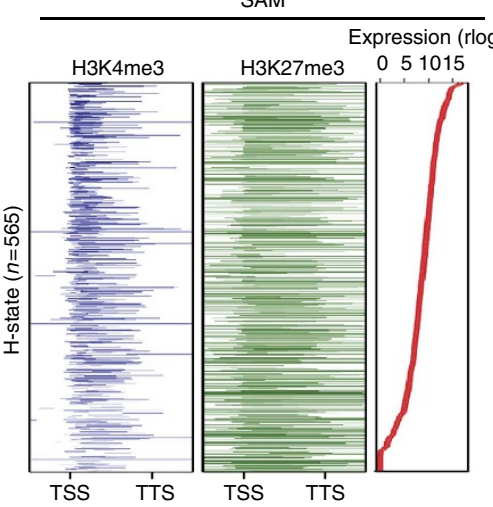

d
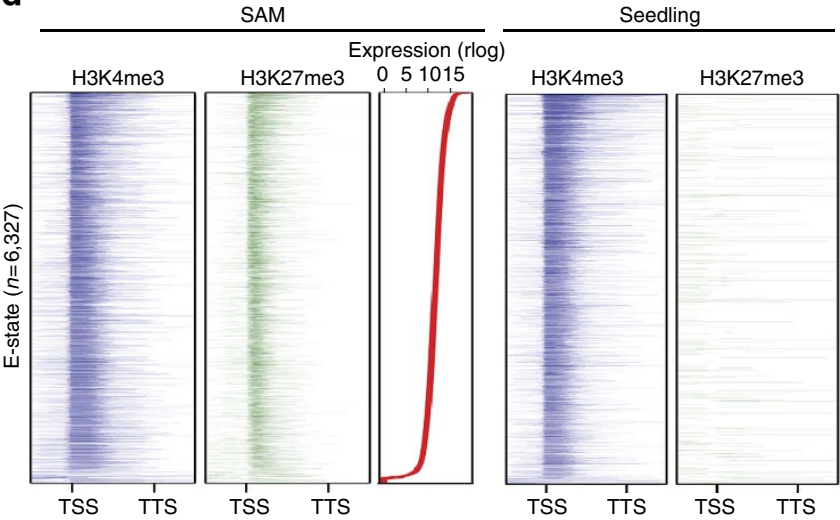

Figure $\mathbf{6}$ | Enrichment of $\mathbf{H}$ - and E- states at the SAM. (a,b) Overlap of genes in $\mathrm{H}$-state (a) and $\mathrm{E}$-state (b) detected in $\mathrm{O}$ LD SAM and in wholeseedling samples. (c,d) Distribution of H3K4me3 and H3K27me3 marks across the gene body ( $\pm 1 \mathrm{~kb})$ of genes in $\mathrm{H}$-state (c) and E-state (d) in 0 LD SAM (left) and the whole seedlings (right). Genes were ordered by expression in the meristem samples (red lines).

Our analysis of the dynamic changes of two histone modifications, $\mathrm{H} 3 \mathrm{~K} 4 \mathrm{me} 3$ and $\mathrm{H} 3 \mathrm{~K} 27 \mathrm{me} 3$, and their correlation with transcription during the transition to flowering has identified two unusual chromatin states at the SAM. Both of these chromatin states, to which we refer as $\mathrm{H}$ - and E-state, display high levels of H3K27me3 at the TSS. In particular the E-state, which is characterized by narrow peaks of H3K27me3 within wider regions of $\mathrm{H} 3 \mathrm{~K} 4 \mathrm{me} 3$, and which we observe at the four time points analysed in total on more than 9,000 genes stands apart, as $\mathrm{H} 3 \mathrm{~K} 27 \mathrm{me} 3$ at the TSS has previously been only observed at much lower frequency ${ }^{33}$. Importantly, the E-state might be rather specific to the undifferentiated cells at the SAM as it was not apparent in another INTACT study, which had analysed hair and non-hair cell in the root epidermis ${ }^{17}$, and was also largely absent from the whole-seedling data ${ }^{33}$. Together, these findings provide evidence for SAM-specific chromatin states at the TSS, emphasizing the value of and need for tissue- (or even cell type-) specific epigenomic analyses.

Genes in E-state were significantly enriched for GO categories related to ribosome function and translation. Similarly, ribosomal proteins and translation initiation factors were also overrepresented among the genes whose expression decreased during flowering. Several examples demonstrate the importance of regulation of translation in animal and plant development ${ }^{42}$, and some ribosomal proteins have even been suggested to possess a non-ribosome function ${ }^{42,44}$. In Arabidopsis thaliana, many ribosomal proteins, including $P G Y 1, P G Y 2$ and $R P L 23 a A$, are preferentially expressed in meristems and young dividing tissues, and loss of function of these genes causes pleiotropic developmental defects ${ }^{29,30}$. Taken together, our results could suggest that control of translational activity might contribute to the regulation of fate transition of the SAM. Finally, RNA-binding proteins that mediate $18 \mathrm{~S}$ rRNA biogenesis have been shown to be preferentially expressed in mouse embryonic stem cells (ESC) where they play a critical role in sustaining the protein levels of labile pluripotency factors and are required for efficient reprogramming of induced pluripotent stem cells ${ }^{45}$. It is tempting to speculate that, similar to the situation in animal ESCs, control of global translation rates could be involved in maintaining pluripotency of the plant meristem.

Reprogramming of epigenetic states is critical for initial establishment and subsequent maintenance of lineage-specific transcriptional programs during the differentiation of cells. In agreement with the important role of transcriptional reprogramming, we have identified 226 TFs, many of which are known to play important roles in regulation flowering and other developmental processes, among the genes that display significant changes in $\mathrm{H} 3 \mathrm{~K} 4 \mathrm{me} 3$ at the SAM during the transition to flowering (Supplementary Fig. 7). This includes TFs related to circadian clock function such as REVEILLE $(R V E 1)^{46}, C C A 1$ and $L H Y$, whose expression significantly change together with H3K4me3 changes (Supplementary Fig. 7) and that all affect long-day photoperiodism and flowering. However, for most of these TFs we observe only small changes in expression (Supplementary Fig. 11), including the important flowering time regulator $S O C 1$, which are not statistically significant after correcting for multiple testing (Supplementary Data 2). Moreover, a few of these TFs do not change in their expression, for example, the flowering time repressor SCHNARCHZAPFEN $(S N Z)^{47}$, whose $\mathrm{H} 3 \mathrm{~K} 4 \mathrm{me} 3$ level was significantly decreased at the TSS (Figs 2e and $4 \mathrm{l}$ and Supplementary Data 2). Our gene expression data and qRT-PCR experiments support the notion that expression of developmentally regulated genes in the SAM is usually rather low. However, if expression of at least some of these genes were confined to a small sub-population of cells in the SAM this could also result in an underestimation of their actual expression changes. Also we cannot completely rule out the possibility that our low-input RNA-seq method, which involves 2-step cDNA amplification, limits our ability to detect changes of weakly expressed genes. Nevertheless, our results suggest that changes in $\mathrm{H} 3 \mathrm{~K} 4 \mathrm{me} 3$ are important early events in the regulation of (and might even precede) changes in gene expression at the SAM during the initiation of flowering.

Other known flowering time genes do change significantly in their expression at the SAM in response to LD. One example is JMJ30, which encodes a Jumonji-C domain-containing H3K36me2 demethylase ${ }^{25}$. One of the confirmed targets of JMJ30 is FT. However, since FT is not expressed at the SAM, JMJ30 likely exerts its effect on flowering at the SAM through other targets. JMJ30 has also been shown to be under direct transcriptional control of CCA1 and LHY, two key components of the central circadian oscillator, which directly bind to JMJ30 promoter to repress its expression ${ }^{48}$. Loss of $J M J 30$ function in turn has been shown to affect the circadian clock ${ }^{48}$. Another example is the CCAAT-motif binding TF, NF-YA4, which has 
a

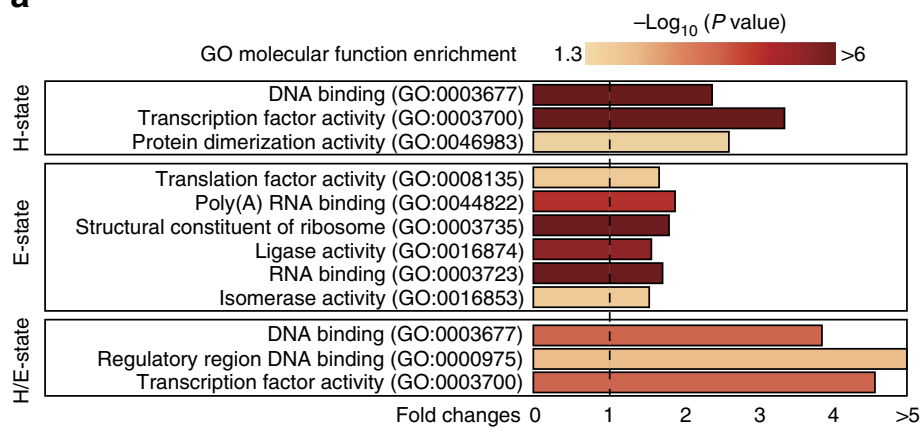

b

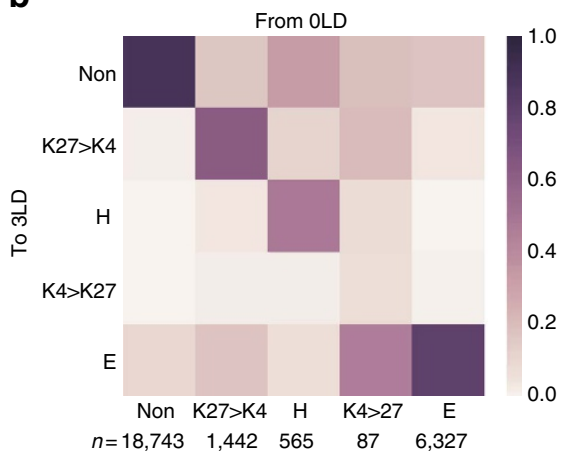

Figure 7 | H- and E-state genes at the SAM during floral transition. (a) Molecular GO functions enriched for $\mathrm{H}$-state, E-state and $\mathrm{H} / \mathrm{E}$-state genes. Only significantly enriched $\mathrm{GO}$ terms $(P<0.05$, fold-change $>1.5)$ are reported. (b) Matrix of chromatin state transitions from $0-3$ LDs for non-overlapping (Non), H-state, and E-state genes, and genes with partial overlapping H3K27me3-H3K4me3 marks (K27>K4; K4>K27). Colours indicate the fraction of genes in a given category on $3 \mathrm{LD}$, starting from the $0 \mathrm{LD}$ sample ( $n=$ number of genes in each category in the 0 LD sample).

been shown to mediate $\mathrm{H} 3 \mathrm{~K} 27 \mathrm{me} 3$ demethylation of the SOC1 promoter to facilitate flowering ${ }^{49}$. In our analysis, expression of NF-YA4 was significantly decreased at the SAM, however, no changes were observed for H3K27me3 on SOC1, which was broadly covered from TSS to TTS throughout the entire experiment (Fig. 4k). Instead, our data suggest that activation of SOC1 expression is accompanied by increasing $\mathrm{H} 3 \mathrm{~K} 4 \mathrm{me} 3$ at the TSS (Fig. 4k), similar to what has been described for FT, which also is regulated largely independently from H3K27me3 (ref. 50), but strongly affected by $\mathrm{H} 3 \mathrm{~K} 4 \mathrm{me} 3$ modification level at the $\mathrm{TSS}^{51}$.

Interestingly, increased expression of KNAT2, KNAT6 and $P T L$, which are expressed at the boundary between meristem and emerging primordia where pluripotent meristem cells start to differentiate ${ }^{35-37}$, is associated with both increasing $\mathrm{H} 3 \mathrm{~K} 4 \mathrm{me} 3$ signals and decreasing H3K27me3 coverage. Taking together our results suggest that $\mathrm{H} 3 \mathrm{~K} 4 \mathrm{me} 3$ has an important role in epigenetic regulated transcription changes at the SAM, whereas the role of $\mathrm{H} 3 \mathrm{~K} 27 \mathrm{me} 3$ during the early stages of the floral transition is limited.

It remains unclear at this point whether the overlapping $\mathrm{H} 3 \mathrm{~K} 4 \mathrm{me} 3$ and $\mathrm{H} 3 \mathrm{~K} 27 \mathrm{me} 3$ signals we observe in our data are a reflection of the sampled SAM tissue, which is composed of different cell types in different stages of differentiation, or if at least some of the peaks are caused by true bivalent marks, that is labelling of a given histone molecule by different modifications. Co-occurrence of $\mathrm{H} 3 \mathrm{~K} 27 \mathrm{me} 3$ and $\mathrm{H} 3 \mathrm{~K} 4 \mathrm{me} 3$ has previously been reported, both on individual genes, such as FT and FLC, as well as in whole-genome analyses ${ }^{17,33,34}$. These analyses were mostly performed on whole seedlings and the detection of $\mathrm{H} 3 \mathrm{~K} 27 \mathrm{me} 3$ and $\mathrm{H} 3 \mathrm{~K} 4 \mathrm{me} 3$ in a specific region of the genome therefore most likely reflects differences in the epigenetic makeup of different cell types rather than bivalent epigenetic marks. However, bivalency does exist in plants as demonstrated by sequential ChIP-PCR analysis in the selected gene loci including $F T$ and $F L C^{33,34}$. At the SAM the $F T$ locus was marked broadly with H3K27me3 from TSS to TTS throughout the entire experiment (Fig. 4c) and the FT gene was transcriptionally silent (Supplementary Data 1). The FLC locus was in $\mathrm{H}$-state $(0 \mathrm{LD}$ and $1 \mathrm{LD}$ ) or partial overlapping state (H3K27me3 > H3K4me3; 2 LD and 3 LD; Fig. 4h, Supplementary Data 9), and no changes were observed for $\mathrm{H} 3 \mathrm{~K} 4 \mathrm{me} 3$ and H3K27me3 signals nor FLC expression.

In ESC, bivalency has been suggested to poise transcription of important differentially regulated genes, but suitable in vivo genetic models to probe functions of bivalency in developing organisms are currently missing ${ }^{52}$. Comparisons between ESCs and differentiated cells suggested that bivalent domains tend to resolve during differentiation, which is associated with changes in gene expression ${ }^{53}$. Since the SAM consists of stem cells and only partially differentiated cells, our finding could point towards commonalities in the characteristics of $\mathrm{H} 3 \mathrm{~K} 4 \mathrm{me} 3$ and $\mathrm{H} 3 \mathrm{~K} 27 \mathrm{me} 3$ between plants and animals. In our study the $\mathrm{H}$-state is reminiscent of the bivalent situation in ESCs, and TFs were overrepresented among the genes that were in H-state. Unfortunately, sequential ChIP-seq (or -PCR) is at this point not really feasible given the very limited amount of starting material available from INTACT, so that the nature of the overlapping $\mathrm{H} 3 \mathrm{~K} 4 \mathrm{me} 3$ and $\mathrm{H} 3 \mathrm{~K} 27 \mathrm{me} 3$ signals at the SAM cannot be answered conclusively at this time.

\section{Methods}

Oligonucleotides. Sequences of oligonucleotide primers used in this work are listed in Supplementary Table 1.

Plant growth conditions. Arabidopsis thaliana accession Col-0 was used in this work. To synchronize germination and floral transition, all seeds were stratified before sowing in $0.1 \%$ agar at $4{ }^{\circ} \mathrm{C}$ for 3 day in the dark. Seeds were sown on soil and all plants were grown in growth chambers in a controlled environment, at $23^{\circ} \mathrm{C}$ with $65 \%$ relative humidity. The light condition was constructed by a mixture of Cool White and Gro-Lux Wide Spectrum fluorescent lights, with a fluence rate of $125-175 \mu \mathrm{mol} \mathrm{m}^{-2} \mathrm{~s}^{-1}$. LDs were $16 \mathrm{~h}$ of light and $8 \mathrm{~h}$ of dark, and SDs were $8 \mathrm{~h}$ of light and $16 \mathrm{~h}$ of dark. In shift experiments for floral induction plants were grown in SDs for 21 days before shifting to LDs.

Plasmid constructs and the SAM-specific INTACT reporter line. The E. coli biotin ligase (BirA) gene was cloned in a modified Gateway entry plasmid pCR8/ GW-TOPO (pDP084), and subsequently recombined into a pGREEN-IIS based destination vector ( $\mathrm{pFK} 273$ ) that harbours the Arabidopsis thaliana $p U B Q 10$ (At4g05320) promoter, using a modified Gateway recombination system ${ }^{54}$. The pUBQ10::BirA construct (pDP085) was transformed into Col-0 plants making use of Agrobacterium tumefaciens strain ASE and the floral dip method ${ }^{55}$.

Homozygous $p U B Q 10:: B i r A$ lines were identified by selective germination on soil watered with $0.1 \%$ glufosinate (BASTA). For INTACT, a modified red nuclear envelope-targeting protein (RedNTF), consisting of the WPP domain (amino acids 1-111) of the Arabidopsis thaliana RAN GTPASE ACTIVATING PROTEIN 1 (RanGAP1; At3g63130) at the $\mathrm{N}$ terminus, followed by red fluorescent protein (mCherry) for visualization, and the biotin ligase recognition peptide at the $\mathrm{C}$ terminus was prepared by PCR using GL2p:NTF ${ }^{17}$ as template. The RedNTF open reading frame was cloned in a modified Gateway entry plasmid (pJLSmart) to create pYY1204. For expression of RedNTF at the SAM, two pGREEN-IIS based destination vectors were used. pYY364 consisted of a 1,393 bp promoter fragment of At3g59270, which was cloned in front of the attR1-attR2 GateWay recombination cassette, followed by the At3g59270 terminator sequence (1,404 bp) and drives expression in the entire meristem except for the stem cells. pFK321 consisted of a 1,444 bp promoter sequence of CLAVATA 3 (CLV3) in the front of the attR1-attR2 cassette, followed by the CLV3 terminator sequence $(1,253 \mathrm{bp})^{56}$ and drives expression of the reporter in the stem cell population at the shoot meristem. pAt3g59270::RedNTF::tAt3g59270 construct (pYY1208) and $p C L V 3 p:: R e d N T F:: t C L V 3$ construct (pYY1301) were generated by recombination of 
pYY1204 into pYY364 and pFK321, respectively. pYY1208 and pYY1301 were subsequently transformed into homozygous $P U B Q 10:: B i r A$ plants to generate CLV3 and At3g59270 RedNTF reporter lines. Single insertion lines for the individual RedNTF reporters were identified by selective germination of T2 seeds on $1 / 2 \mathrm{MS}$ agar plates containing $50 \mu \mathrm{g} \mathrm{ml}^{-1}$ of kanamycin. INTACT lines that carried both reporters were created by genetic crossings and double-homozygous lines that expressed RedNTF in the meristem were selected by PCR-based genotyping and used in subsequent experiments. PCR reactions were performed using Phusion polymerase or Taq polymerase (New England Biolabs), and all constructs were verified by Sanger sequencing after cloning.

In situ RNA hybridization and Immunohistochemistry. The digoxigenin-labeled anti-sense RNA probe to APETALA 1 (AP1) mRNA was prepared using a published plasmid ${ }^{57}$. Shoot apices from SD-grown plants (21 days) and plants shifted to LD for 1,2 or 3 days were dissected and fixed in formaldehyde/acetic acid/ethanol (3.7\%/5\%/50\%). Paraplast-embedded material was sectioned to $8 \mu \mathrm{m}$ thickness and RNA in situ hybridization was performed as described ${ }^{57}$. To detect in vivo biotinylation by immunohistochemistry sections were dewaxed and rehydrated before applying 1:2,000 diluted streptavidin alkaline phosphatase (Promega) in 1x TBS $(0.1 \%$ Triton X-100). Permeabilization and immunohistochemical staining were carried out at the room temperature for $1 \mathrm{~h} 30 \mathrm{~min}$. The signal was developed by a colour reaction using NBT-BCIP solution (Roche).

Microscopy. A ZEISS Axioplan 2 imaging system was used for imaging the histological sections and for examining the quality and quantity of purified nuclei after each INTACT experiments using DAPI and mCherry filters. In addition, nuclei isolated from the INTACT reporter line were also imaged using a Zeiss LSM510 confocal laser-scanning microscope.

INTACT purification of SAM nuclei. For each biological replicate, $\sim 6,000$ INTACT reporter plants were grown in SDs for 21 days before shifting to LDs. The $0 \mathrm{LD}, 1 \mathrm{LD}, 2 \mathrm{LD}$ and $3 \mathrm{LD}$ samples were collected on the 21 st day in SDs and 1,2 and 3 days after the shift to LD. For each sample, the shoot apical region was manually dissected from $\sim 1,300$ INTACT reporter plants and frozen immediately in Eppendorf tubes suspended in liquid nitrogen. Each sample collection took about $3 \mathrm{~h}$ from ZT5 to 8 and samples were stored at $-80^{\circ} \mathrm{C}$ before processing. The INTACT experiments were carried out as described ${ }^{15}$ with the following modifications. Briefly, the 1,300 frozen apices were homogenized to a fine powder with mortar and pestle pre-chilled in liquid nitrogen. Approximately $1 / 3$ of the powder was suspended in $10 \mathrm{ml}$ of ice-cold nuclei purification buffer (NPB: $20 \mathrm{mM}$ MOPS (pH 7), $40 \mathrm{mM} \mathrm{NaCl}, 90 \mathrm{mM} \mathrm{KCl}, 2 \mathrm{mM}$ EDTA, $0.5 \mathrm{mM}$ EGTA, $0.5 \mathrm{mM}$ spermidine, $0.2 \mathrm{mM}$ spermine, $1 \times$ Complete protease Inhibitors (Roche)), for isolating non-fixed nuclei by INTACT for transcriptome analysis by RNA-seq. The remaining approximately $2 / 3$ of the ground tissue was suspended in $10 \mathrm{ml}$ of icecold NPB buffer containing $1 \%$ (vol/vol) formaldehyde (NPBf) and placed under vacuum at RT for $10 \mathrm{~min}$ to cross-link proteins to DNAs. The cross-linking was stopped by adding $2 \mathrm{M}$ glycine to a final concentration of $125 \mathrm{mM}$ and incubated for an additional $5 \mathrm{~min}$ under vacuum. The fixed nuclei from the SAM were isolated by INTACT and subsequently used to analyze histone modifications by ChIP-seq. Nuclear suspensions were filtered through $40 \mu \mathrm{m}$ cell strainers

(BD Falcon) and pelleted at $1,000 \mathrm{~g}$ for $5 \mathrm{~min}$ at $4{ }^{\circ} \mathrm{C}$. Nuclei were washed with $1 \mathrm{ml}$ of NPB, pelleted again by centrifugation and finally resuspended in $1 \mathrm{ml}$ of NPB. For each sample $25 \mu \mathrm{l}$ of M-280 streptavidin Dynabeads (Invitrogen) were washed with $1 \mathrm{ml}$ of NPB, pelleted at $3,500 \mathrm{~g}$ for $2 \mathrm{~min}$ at $4{ }^{\circ} \mathrm{C}$, resuspended in $25 \mu \mathrm{lNPB}$, and finally added to the nuclear suspensions. The mixture was rotated at $4{ }^{\circ} \mathrm{C}$ for $1 \mathrm{~h}$ to allow binding of biotinylated nuclei to the streptavidin-coated beads. The setup of the INTACT experiments consisted of an OctoMACS TM Seperator (Miltenyi Biotec) placed on ice with BSA-coated $0.6 \mathrm{ml}$ tubes tightly fitted into the grooves. After binding the $1 \mathrm{ml}$ of nuclei-bead suspension was diluted to $10 \mathrm{ml}$ with ice-cold NPB. The $10 \mathrm{ml}$ nuclei-beads suspension of each sample was successively transferred into the $0.6-\mathrm{ml}$ tube fitted in to the OctoMACS TM Seperator, with 3 min intervals in between aliquots to allow nuclei-beads to be captured by the magnet. After each aliquot, the liquid containing un-bound nuclei was removed using a needle and syringe and discarded, until all of the suspension had been processed. The purified nuclei were washed two times with NPB buffer. For each wash step the tubes were carefully removed from the magnets and the magnetic beads were gently resuspended in $500 \mu \mathrm{l}$ ice-cold NPB and transferred into fresh tubes, which were left at the magnet for $3 \mathrm{~min}$, before the wash buffer was removed using a syringe with a fresh needle and discarded. Finally the purity and the yield of intact nuclei was estimated by microscopy as described ${ }^{15}$ and samples in which INTACT nuclei were enriched to $>90 \%$ purity were retained and stored at

$-80^{\circ} \mathrm{C}$ before subsequent experiments.

RNA extraction and double-strand cDNA amplification. Total nuclear RNA was extracted from $\sim 10,000$ non-fixed nuclei of each sample using the RNeasy Micro Kit (Qiagen) following the manufacturers instructions. Contaminating genomic DNA was removed from the RNA samples by treatment with DNase I ( $0.05 \mathrm{U}$ per $\mu \mathrm{l})$ for $30 \mathrm{~min}$ at $37^{\circ} \mathrm{C}$ (Thermo Scientific) before the RNA was purified a second time using the RNeasy Micro Kit (Qiagen). The concentration of the nuclear RNA was determined using the RNA 6,000 Pico Kit (Agilent). The double-stranded cDNA was synthesized from $\sim 500 \mathrm{pg}$ RNA by linear amplification using the SMARTer Ultra Low Input RNA for Illumina Sequencing-HV kit (Clontech) according to the manufacturer's instructions. The concentration and yield of the amplified cDNA was determined using the High Sensitivity DNA Kit (Agilent).

Quantitative PCR. The enrichment of the meristematic genes in the isolated nuclei after the INTACT purification was examined by semi-quantitative PCR. $\sim 100 \mathrm{pg}$ amplified double-stranded cDNA prepared from the input and purified 3 LD samples was used in PCR reactions to examine the expression of STM, CLV3 and $F D$ genes (Supplementary Fig. 2) using gene-specific primers (Supplementary Table 1). After 40 cycles the PCR products were separated on a $1.5 \%$ agarose gel followed by ethylbromide staining to visualize DNA. Real-time qPCR of SOC1 and AGL24 was performed from $\sim 100 \mathrm{pg}$ amplified double-stranded cDNA, using the Platinum SYBR Green qPCR Supermix-UDG (Invitrogen, Life Technologies) and gene-specific oligonucleotides (Supplementary Table 1) on BioRad C1000 Touch Thermal Cycler. The relative expression values were normalized over the mean expression value of $0 \mathrm{LD}$ samples and calculated by the $\Delta \Delta C \mathrm{t}$ method using TUB2 as control. Because amplification signals from the 0 LD SOC1 samples did not cross the detection threshold, the $C t$ values was set to 40 , the number of cycles used in the PCR programme, to be able to conservatively estimate the differences in expression. Two biological replications with 3 technical replications per sample were used for $\mathrm{qPCR}$ analysis.

RNA sequencing. For transcriptome profiling, 1-2 ng of amplified double-stran ded cDNA was sheared to $200-500$ bp fragments in water using a focused ultrasonicator (Covaris S2 system), using microtubes at a power of ' $10 \mathrm{dc}, 5 \mathrm{i}, 200 \mathrm{cpb}$ fs' for $40 \mathrm{~s}$. Subsequently the Low Input Library Prep Kit (Clontech) was used for preparation of sequencing libraries. Libraries were validated using the High Sensitivity DNA Kit (Agilent), and sequencing was performed on an Illumina Hiseq 2,000 system. Approximately 30 million $2 \times 101$ base-pair paired-end reads that passed the Illumina quality control were collected for each sample in four biological replications (Supplementary Table 2).

Chromatin immunoprecipitation and sequencing. For ChIP-seq experiments using INTACT-purified nuclei, chromatin was prepared from $\sim 10,000$ purified fixed nuclei per sample as described above. ChIP was performed as described ${ }^{15}$ with the following modifications: chromatin was sheared to $100-500 \mathrm{bp}$ fragments in $200 \mu \mathrm{l}$ lysis buffer (50 mM Tris, $\mathrm{pH}$ 8.0, $10 \mathrm{mM}$ EDTA, $1 \%$ SDS, $1 \times$ complete protease inhibitors (Roche)) using a focused ultrasonicator (Covaris S2 system), using microtubes at a power of ' $10 \mathrm{dc}, 5 \mathrm{i}, 200 \mathrm{cpb}$ fs' for $3 \mathrm{~min}$. The sheared chromatin was divided into three aliquots at the ratio of 1:2:3 for immunohybridization with $0.5 \mu \mathrm{l}$ anti-H3 antibody (Millipore, Cat.17-10254, Lot. 2051404), $1.5 \mu \mathrm{l}$ anti-H3K4me3 antibody (Millipore, Cat.17-614, Lot.1973237), and $2.5 \mu \mathrm{l}$ anti-H3K27me3 antibody (Active Motif, Cat. 39155, Lot. 25812014), respectively. The specificity and sensitivity of the antibodies was verified by western blot using nuclear protein extracts as described ${ }^{58}$, and by dot-blot analysis using peptides containing non-, mono-, di- and tri-methylated versions of the respective residues (Abcam, ab2623, ab2903, ab1340, ab7768, ab1342 and ab1780-1782), according to a protocol provided by the manufacturer. Approximately $50 \mathrm{pg}$ of ChIP DNA per sample was isolated using the MinElute Reaction Cleanup Kit (Qiagen). The ChIP-seq libraries were prepared using the ThruPlex-FD Prep Kit (Rubicon Genomics), and linear amplification was monitored by quantitative PCR including SYBR Green I (Invitrogen). Libraries were quantified using the KAPA Library Quantification Kit (KAPABIOSYSTEMS), and further evaluated on a high sensitivity DNA chip (Agilent).

For control ChIP-seq experiments using 3-weeks-old LD-grown seedlings, chromatin was prepared from $\sim 2 \mathrm{~g}$ of fresh material as described ${ }^{59}$. The ChIP experiments were performed as described above and libraries were prepared from $\sim 5 \mathrm{ng}$ of ChIP DNA using the TruSeq ChIP Library Prep Kit (Illumina). Libraries were quantified on a high sensitivity DNA chip (Agilent).

Libraries were sequenced on Illumina MiSeq, HiSeq 2,000 and HiSeq 3,000 systems for collecting 50 base-pair single-end or 150 base-pair paired-end reads to high sequencing depth ${ }^{60}$ (Supplementary Table 2). Only Read 1 of 150 base-pair paired-end reads were used and further trimmed to 50 base pair. Uniquely mapped 50 base-pair single-end reads were used for ChIP-seq analyses.

RNA-seq data analysis. Next generation sequencing (NGS) reads were mapped to Arabidopsis thaliana reference transcriptome TAIR10 ver. 24, with ribosomal RNA regions (2:3471-9557; 3:14,197,350-14,203,988) masked, using TopHat 2.0.13 (no-mixed alignments; up to 20 secondary alignments; no novel junctions) ${ }^{61}$ Counts of NGS reads covering transcripts were computed using the function summarizeOverlaps ${ }^{62}$ in R. Expressed genes were defined as those having the value of FPKM $>1$ at least at one time point. Read counts were submitted to differential gene expression analysis in Deseq2 (default parameters, FDR $<0.05)^{63}$. Regularized logarithms of read count computed by Deseq2, denoted by rlog, were used for the analysis of relationships between gene expression level and histone modifications signal. 
Histone modification data analysis. NGS reads were mapped to Arabidopsis thaliana reference genome TAIR10 ver. 24 using Bowtie2 2.2.4 (minimum alignment score -12.5 ; uniquely mapped reads only) ${ }^{64}$. The sets of aligned H3K27me3 and $\mathrm{H} 3$ reads were down-sampled to minimum number of reads, respectively, for peak-calling from the same sequencing depth in all samples ${ }^{65}$. Significant enrichment regions in $\mathrm{H} 3 \mathrm{~K} 4 \mathrm{me} 3$ and $\mathrm{H} 3 \mathrm{~K} 27 \mathrm{me} 3$ samples, relative to $\mathrm{H} 3$ control samples, were identified using MACS2 2.1.0 (default settings for H3K4me3$\mathrm{FDR}<0.05$; broad-peaks mode for H3K27me3-FDR $<0.1)^{66,67}$. Regions with signal level measured by FPKM(ChIP)/FPKM(H3 control) differing between biological replications by more than 2 s.d. were filtered out. Consensus regions found as a sum of regions from two replications were taken as histone modification marks. Differential modification analysis for $\mathrm{H} 3 \mathrm{~K} 4 \mathrm{me} 3$ and $\mathrm{H} 3 \mathrm{~K} 27 \mathrm{me} 3$ marks was performed using DiffBind ${ }^{68}$ (Bioconductor 3.2; minoverlap $=2$; FDR $<0.01$ ). The modification marks were intersected with annotated genes to obtain lists of genes with specific coverage by $\mathrm{H} 3 \mathrm{~K} 4 \mathrm{me} 3$ and $\mathrm{H} 3 \mathrm{~K} 27 \mathrm{me} 3$. Spearman rank correlation coefficients were computed and tested for significance in Genstat 18 (ref. 69).

GO term analysis. AmiGO 2 versions 2.3.2 and 2.4.24 (ref. 70) were used for GO analysis to assess the over- and underrepresentation in biological processes of significantly differentially-H3K4me3-modified genes and molecular functions of $\mathrm{H}$-state and E-state genes. $P$ values were corrected for multiple hypothesis testing (Bonferroni correction), and only $P<0.05$ were considered as significant. To reduce the complexity redundant child terms based on GO hierarchy were removed from the reports.

Data availability. ChIP-seq and RNA-seq data have been deposited with ArrayExpress database (www.ebi.ac.uk/arrayexpress), accession numbers E-MTAB-4680, E-MTAB-4684 and E-MTAB-5130. The authors declare that all other data supporting the findings of this study are available within the manuscript and its supplementary files or are available from the corresponding author upon request.

\section{References}

1. Weigel, D. \& Jürgens, G. Stem cells that make stems. Nature 415, 751-754 (2002).

2. Wilczek, A. M. et al. Effects of genetic perturbation on seasonal life history plasticity. Science 323, 930-934 (2009).

3. Kwiatkowska, D. Flowering and apical meristem growth dynamics. J. Exp. Bot. 59, 187-201 (2008).

4. de Montaigu, A. et al. Natural diversity in daily rhythms of gene expression contributes to phenotypic variation. Proc. Natl Acad. Sci. USA 112, 905-910 (2015).

5. Corbesier, L. et al. FT protein movement contributes to long-distance signaling in floral induction of Arabidopsis. Science 316, 1030-1033 (2007).

6. Yamaguchi, A., Kobayashi, Y., Goto, K., Abe, M. \& Araki, T. TWIN SISTER OF FT (TSF) acts as a floral pathway integrator redundantly with FT. Plant Cell Physiol. 46, 1175-1189 (2005).

7. Immink, R. G. et al. Characterization of SOC1's central role in flowering by the identification of its upstream and downstream regulators. Plant Physiol. 160, 433-449 (2012).

8. Schmid, M. et al. A gene expression map of Arabidopsis thaliana development. Nat. Genet. 37, 501-506 (2005).

9. Torti, S. et al. Analysis of the Arabidopsis shoot meristem transcriptome during floral transition identifies distinct regulatory patterns and a leucine-rich repeat protein that promotes flowering. Plant Cell 24, 444-462 (2012).

10. Klepikova, A. V., Logacheva, M. D., Dmitriev, S. E. \& Penin, A. A. RNA-seq analysis of an apical meristem time series reveals a critical point in Arabidopsis thaliana flower initiation. BMC Genomics 16, 466 (2015).

11. Jeong, H. J., Yang, J., Yi, J. \& An, G. Controlling flowering time by histone methylation and acetylation in arabidopsis and rice. J. Plant Biol. 58, 203-210 (2015).

12. Wang, Y., Gu, X., Yuan, W., Schmitz, R. J. \& He, Y. Photoperiodic control of the floral transition through a distinct polycomb repressive complex. Dev. Cell 28, 727-736 (2014).

13. Yang, W., Jiang, D., Jiang, J. \& He, Y. A plant-specific histone H3 lysine 4 demethylase represses the floral transition in Arabidopsis. Plant J. 62, 663-673 (2010).

14. Yang, H., Howard, M. \& Dean, C. Physical coupling of activation and derepression activities to maintain an active transcriptional state at FLC. Proc. Natl Acad. Sci. USA 113, 9369-9374 (2016).

15. Deal, R. B. \& Henikoff, S. The INTACT method for cell type-specific gene expression and chromatin profiling in Arabidopsis thaliana. Nat. Protoc. 6, 56-68 (2010).

16. Roudier, F. et al. Integrative epigenomic mapping defines four main chromatin states in Arabidopsis. EMBO J. 30, 1928-1938 (2011).

17. Deal, R. B. \& Henikoff, S. A simple method for gene expression and chromatin profiling of individual cell types within a tissue. Dev. Cell 18, 1030-1040 (2010).
18. Daum, G., Medzihradszky, A., Suzaki, T. \& Lohmann, J. U. A mechanistic framework for noncell autonomous stem cell induction in Arabidopsis. Proc. Natl Acad. Sci. USA 111, 14619-14624 (2014).

19. Abe, M. et al. FD, a bZIP protein mediating signals from the floral pathway integrator FT at the shoot apex. Science 309, 1052-1056 (2005).

20. Schmid, M. et al. Dissection of floral induction pathways using global expression analysis. Development 130, 6001-6012 (2003).

21. Tsukaya, H. Leaf Development. The Arabidopsis Book 11, e0163 (2013).

22. McClung, C. R. Wheels within wheels: new transcriptional feedback loops in the Arabidopsis circadian clock. F1000Prime Rep. 6, 2 (2014).

23. Michael, T. P. et al. Network discovery pipeline elucidates conserved time-ofday-specific cis-regulatory modules. PLoS Genet. 4, e14 (2008).

24. Wenkel, S. et al. CONSTANS and the CCAAT box binding complex share a functionally important domain and interact to regulate flowering of Arabidopsis. Plant Cell 18, 2971-2984 (2006).

25. Yan, Y. et al. A MYB-domain protein EFM mediates flowering responses to environmental cues in Arabidopsis. Dev. Cell 30, 437-448 (2014).

26. Ishiguro, S. SHEPHERD is the Arabidopsis GRP94 responsible for the formation of functional CLAVATA proteins. EMBO J. 21, 898-908 (2002).

27. Bisanz, C. et al. The Arabidopsis nuclear DAL gene encodes a chloroplast protein which is required for the maturation of the plastid ribosomal RNAs and is essential for chloroplast differentiation. Plant Mol. Biol. 51, 651-663 (2003).

28. Ito, T., Kim, G.-T. \& Shinozaki, K. Disruption of an Arabidopsis cytoplasmic ribosomal protein S13-homologous gene by transposon-mediated mutagenesis causes aberrant growth and development. Plant J. 22, 257-264 (2000).

29. Degenhardt, R. F. \& Bonham-Smith, P. C. Arabidopsis ribosomal proteins RPL23aA and RPL23aB are differentially targeted to the nucleolus and are disparately required for normal development. Plant Physiol. 147, 128-142 (2008).

30. Pinon, V. et al. Three PIGGYBACK genes that specifically influence leaf patterning encode ribosomal proteins. Development 135, 1315-1324 (2008).

31. Lafos, M. et al. Dynamic regulation of $\mathrm{H} 3 \mathrm{~K} 27$ trimethylation during Arabidopsis differentiation. PLoS Genet. 7, e1002040 (2011).

32. Brusslan, J. A. et al. Genome-wide evaluation of histone methylation changes associated with leaf senescence in Arabidopsis. PLoS ONE 7, e33151 (2012)

33. Sequeira-Mendes, J. et al. The Functional topography of the Arabidopsis genome is organized in a reduced number of linear motifs of chromatin states. Plant Cell 26, 2351-2366 (2014).

34. Dilkes, B. P., Jiang, D., Wang, Y., Wang, Y. \& He, Y. Repression of FLOWERING LOCUS C and FLOWERING LOCUS T by the Arabidopsis Polycomb repressive complex 2 components. PLoS ONE 3, e3404 (2008).

35. Belles-Boix, E. KNAT6: an Arabidopsis homeobox gene involved in meristem activity and organ separation. Plant Cell 18, 1900-1907 (2006).

36. Brewer, P. B. PETAL LOSS, a trihelix transcription factor gene, regulates perianth architecture in the Arabidopsis flower. Development 131, 4035-4045 (2004).

37. Hepworth, S. R. \& Pautot, V. A. Beyond the divide: boundaries for patterning and stem cell regulation in plants. Front. Plant Sci. 6, 1052 (2015).

38. Weijers, D. et al. An Arabidopsis minute-like phenotype caused by a semidominant mutation in a RIBOSOMAL PROTEIN S5 gene. Development 128, 4289-4299 (2001).

39. Van Lijsebettens, M. et al. An S18 ribosomal protein gene copy at the Arabidopsis PFL locus affects plant development by its specific expression in meristems. EMBO J. 13, 3378-3388 (1994).

40. Vanderhaeghen, R. et al. Leader sequence of a plant ribosomal protein gene with complementarity to the $18 \mathrm{~S}$ rRNA triggers in vitro cap-independent translation. FEBS Lett. 580, 2630-2636 (2006).

41. McIntosh, K. B. \& Bonham-Smith, P. C. The two ribosomal protein L23A genes are differentially transcribed in Arabidopsis thaliana. Genome 48, 443-454 (2005).

42. Byrne, M. E. A role for the ribosome in development. Trends Plant Sci. 14, 512-519 (2009).

43. Kawakatsu, T. et al. Unique cell-type-specific patterns of DNA methylation in the root meristem. Nat. Plants 2, 16058 (2016).

44. Bugner, V., Tecza, A., Gessert, S. \& Kuhl, M. Peter Pan functions independently of its role in ribosome biogenesis during early eye and craniofacial cartilage development in Xenopus laevis. Development 138, 2369-2378 (2011).

45. You, K. T., Park, J. \& Kim, V. N. Role of the small subunit processome in the maintenance of pluripotent stem cells. Genes Dev. 29, 2004-2009 (2015).

46. Rawat, R. et al. REVEILLE1, a Myb-like transcription factor, integrates the circadian clock and auxin pathways. Proc. Natl Acad. Sci. USA 106, 16883-16888 (2009).

47. Mathieu, J., Yant, L. J., Mürdter, F., Küttner, F. \& Schmid, M. Repression of flowering by the miR172 target SMZ. PLoS Biol. 7, e1000148 (2009).

48. Lu, S. X. et al. The Jumonji C domain-containing protein JMJ30 regulates period length in the Arabidopsis circadian clock. Plant Physiol. 155, 906-915 (2010). 
49. Hou, X. et al. Nuclear factor Y-mediated H3K27me3 demethylation of the SOC1 locus orchestrates flowering responses of Arabidopsis. Nat. Commun. 5, 4601 (2014).

50. Farrona, S. et al. Tissue-specific expression of FLOWERING LOCUS T in Arabidopsis is maintained independently of polycomb group protein repression. Plant Cell 23, 3204-3214 (2011).

51. Jeong, J.-H. et al. Repression of FLOWERING LOCUS T chromatin by functionally redundant histone $\mathrm{H} 3$ lysine 4 demethylases in Arabidopsis. PLoS ONE 4, e8033 (2009).

52. Voigt, P., Tee, W. W. \& Reinberg, D. A double take on bivalent promoters. Genes Dev. 27, 1318-1338 (2013).

53. Mikkelsen, T. S. et al. Genome-wide maps of chromatin state in pluripotent and lineage-committed cells. Nature 448, 553-560 (2007).

54. Mathieu, J., Warthmann, N., Küttner, F. \& Schmid, M. Export of FT protein from phloem companion cells is sufficient for floral induction in Arabidopsis. Curr. Biol. 17, 1055-1060 (2007).

55. Clough, S. J. \& Bent, A. F. Floral dip: a simplified method for Agrobacterium-mediated transformation of Arabidopsis thaliana. Plant J. 16, 735-743 (1998).

56. Galvao, V. C., Horrer, D., Kuttner, F. \& Schmid, M. Spatial control of flowering by DELLA proteins in Arabidopsis thaliana. Development 139, 4072-4082 (2012).

57. Wang, J.-W., Czech, B. \& Weigel, D. miR156-regulated SPL transcription factors define an endogenous flowering pathway in Arabidopsis thaliana. Cell 138, 738-749 (2009).

58. Copenhaver, G. P. et al. Polycomb repressive complex 2 controls the embryoto-seedling phase transition. PLoS Genet. 7, e1002014 (2011).

59. Gendrel, A.-V., Lippman, Z., Martienssen, R. \& Colot, V. Profiling histone modification patterns in plants using genomic tiling microarrays. Nat. Methods 2, 213-218 (2005).

60. Sims, D., Sudbery, I., Ilott, N. E., Heger, A. \& Ponting, C. P. Sequencing depth and coverage: key considerations in genomic analyses. Nat. Rev. Genet. 15, 121-132 (2014)

61. Trapnell, C., Pachter, L. \& Salzberg, S. L. TopHat: discovering splice junctions with RNA-Seq. Bioinformatics 25, 1105-1111 (2009).

62. Lawrence, M. et al. Software for computing and annotating genomic ranges. PLoS Comput. Biol. 9, e1003118 (2013).

63. Love, M. I., Huber, W. \& Anders, S. Moderated estimation of fold change and dispersion for RNA-seq data with DESeq2. Genome Biol. 15, 550 (2014).

64. Langmead, B. \& Salzberg, S. L. Fast gapped-read alignment with Bowtie 2. Nat. Methods 9, 357-359 (2012).

65. Jung, Y. L. et al. Impact of sequencing depth in ChIP-seq experiments. Nucleic Acids Res. 42, e74 (2014).

66. Zhang, Y. et al. Model-based analysis of ChIP-Seq (MACS). Genome Biol. 9, R137 (2008).

67. Kellis, M. et al. Defining functional DNA elements in the human genome. Proc. Natl Acad. Sci. USA 111, 6131-6138 (2014).

68. Stark, R. \& Brown, G. D. DiffBind: differential binding analysis of ChIP-seq peak data. Bioconductor http://bioconductor.org/packages/release/bioc/ vignettes/DiffBind/inst/doc/DiffBind.pdf (2011).

69. VSN International. GenStat for Windows18th edn http://www.vsni.co.uk/ (2015).
70. Carbon, S. et al. AmiGO: online access to ontology and annotation data. Bioinformatics 25, 288-289 (2009).

\section{Acknowledgements}

We thank members of the Schmid lab and the Dept. of Molecular Biology at the MPI for Developmental Biology for help with sample collection, C. Lanz, C. Liebig, S. Henz and $\mathrm{N}$. Agarwala for technical assistance, D. Weigel for the comments on the manuscript, and the Poznan Supercomputing and Networking Center for access to their infrastructure for part of the computations. Y.Y. was supported by a postdoctoral fellowship from the MPI for Developmental Biology, Dept. of Molecular Biology. Supported through the ERA-CAPS project 'FlowPlast' by a grant from the Polish National Centre for Research and Development (ERA-CAPS-1/1/2014) to P.K. and the DFG (SCHM 1560/10-1) to M.S., and the MPI for Developmental Biology to M.S. Work on flowering time in the Schmid lab is supported through grants from the Vetenskapsrådet (VR; 2015-04617) and the Knut and Knut and Alice Wallenberg Foundation (KWA; 2016.0025).

\section{Author contributions}

Y.Y. and M.S. designed the experiments. Y.Y. performed most experiments and analysed data; A.S. analysed RNA-seq and ChIP-seq data; M.N. selected homozygous transgenic INTACT line, assisted Y.Y in INTACT experiments and quantified sequencing samples; D.P. prepared UBQ10::BirA and redNTF entry constructs; G.C. performed in situ RNA hybridization to detect $A P 1$ transcript; T.L. generated the meristem-specific expression vector; R.N. participated in data analyses and discussion; P.K. supervised RNA-seq and ChIP-seq analyses; Y.Y. and M.S. supervised the work and wrote the manuscript with input from A.S., P.K. and R.N.

\section{Additional information}

Supplementary Information accompanies this paper at http://www.nature.com/ naturecommunications

Competing interests: The authors declare no competing financial interests.

Reprints and permission information is available online at http://npg.nature.com/ reprintsandpermissions/

How to cite this article: You, Y. et al. Temporal dynamics of gene expression and histone marks at the Arabidopsis shoot meristem during flowering. Nat. Commun. 8, 15120 doi: $10.1038 /$ ncomms15120 (2017).

Publisher's note: Springer Nature remains neutral with regard to jurisdictional claims in published maps and institutional affiliations.

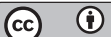

This work is licensed under a Creative Commons Attribution 4.0 International License. The images or other third party material in this article are included in the article's Creative Commons license, unless indicated otherwise in the credit line; if the material is not included under the Creative Commons license, users will need to obtain permission from the license holder to reproduce the material. To view a copy of this license, visit http://creativecommons.org/licenses/by/4.0/

(C) The Author(s) 2017 